\title{
Screening for nonalcoholic fatty liver disease with liver stiffness measurement and its association with chronic kidney disease and cardiovascular complications in patients with type 2 diabetes
}

Short title: NAFLD, liver fibrosis and diabetic vascular complications

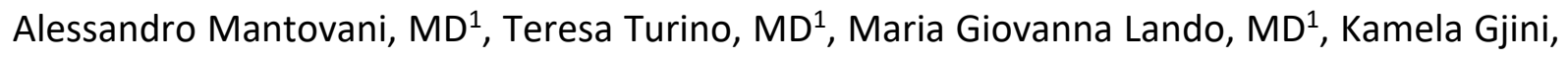
$\mathrm{MD}^{2}$, Christopher D. Byrne, MB BCh, $\mathrm{PhD}^{3,4}$, Chiara Zusi, $\mathrm{PhD}^{1,5}$, Federico Ravaioli, $\mathrm{MD}^{6}$, Antonio Colecchia, MD², Claudio Maffeis, MD5, Gianluca Salvagno, MD ${ }^{7}$, Giuseppe Lippi, MD , Enzo Bonora, $\mathrm{MD}^{1}$, Giovanni Targher, $\mathrm{MD}^{1}$

${ }^{1}$ Division of Endocrinology, Diabetes and Metabolism, Department of Medicine, University Hospital of Verona, Verona, Italy

${ }^{2}$ Gastroenterology Unit, Azienda Ospedaliera Universitaria Integrata of Verona, Verona, Italy ${ }^{3}$ Nutrition and Metabolism, Faculty of Medicine, University of Southampton, UK

${ }^{4}$ Southampton National Institute for Health Research Biomedical Research Centre, University Hospital Southampton, Southampton General Hospital, Southampton SO16 6YD, UK

${ }^{5}$ Pediatric Diabetes and Metabolic Disorders Unit, Department of Surgical Sciences, Dentistry, Pediatrics and Gynaecology, University Hospital of Verona, Verona, Italy

${ }^{6}$ Department of Medical and Surgical Sciences (DIMEC), University Hospital St. OrsolaMalpighi, Alma Mater Studiorum - University of Bologna, Bologna, Italy

${ }^{7}$ Section of Clinical Biochemistry, University and Azienda Ospedaliera Universitaria Integrata of Verona, Verona, Italy

Word count: abstract 250; text 3,791 (excluding title page, abstract, references, figure legends and tables); n. 3 Tables; n. 2 Figures; n. 1 supplementary Table

\author{
Address of correspondence: \\ Prof. Giovanni Targher, MD \\ Section of Endocrinology, Diabetes and Metabolism \\ University and Azienda Ospedaliera Universitaria Integrata \\ Piazzale Stefani, 1 \\ 37126 Verona, Italy \\ Phone: +39/045-8123748 \\ Fax: $+39 / 045-8027314$ \\ E-mail: giovanni.targher@univr.it
}




\section{ABSTRACT}

Aim: Despite the high prevalence and serious clinical implications of nonalcoholic fatty liver disease (NAFLD) in patients with type 2 diabetes (T2DM), it is usually overlooked in routine diabetes care. We explored the proportion of NAFLD and increased liver fibrosis, and the association of liver fibrosis with chronic kidney disease (CKD) or cardiovascular complications in T2DM patients.

Methods: We studied 137 patients with non-insulin-treated T2DM without known liver diseases, consecutively attending our diabetes outpatient service, who underwent liver ultrasonography and liver stiffness measurements (LSM) by vibrated-controlled transient elastography (FibroScan ${ }^{\circledR}$ ).

Results: The proportion of patients with hepatic steatosis on ultrasonography was $73.7 \%$, whereas the proportion with significant liver fibrosis was $17.5 \%$, applying LSM cutoff $\geq 7 \mathrm{kPa}$ or $10.2 \%$ applying LSM cutoff $\geq 8.7 \mathrm{kPa}$. The presence of CKD (i.e., estimated GFR $<60$ $\mathrm{mL} / \mathrm{min} / 1.73 \mathrm{~m}^{2}$ or abnormal albuminuria) increased significantly across LSM tertiles (from $\sim 15 \%$ to $45 \%$ in $1^{\text {st }}$ vs. $3^{\text {rd }}$ tertile). Cardiovascular complications (i.e., prior ischemic heart disease, stroke or permanent atrial fibrillation) also tended to increase across LSM tertiles (from $~ 15 \%$ to $30 \%$ ). After adjustment for established risk factors and potential confounders,

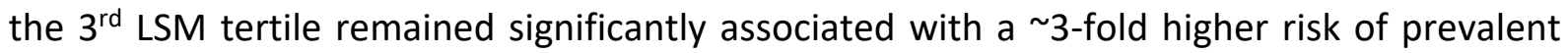
CKD (adjusted-odds 3.28, 95\% Cl 1.22-8.90, $p=0.019$ ), but not with cardiovascular complications.

Conclusion: These results suggest that NAFLD and significant liver fibrosis (assessed by FibroScan ${ }^{\circledR}$ ) are very common in T2DM outpatients without known liver diseases attending a secondary care diabetes service, and that increased liver fibrosis is associated with a higher proportion of chronic vascular complications, especially CKD.

Keywords: CKD; liver fibrosis; NAFLD; type 2 diabetes 


\section{INTRODUCTION}

Nonalcoholic fatty liver disease (NAFLD) is now recognized as the most common chronic liver disease worldwide. This condition occurs in up to a third of adults in the general population in high-income countries and its prevalence is even higher among patients with type 2 diabetes mellitus (T2DM), occurring in up to $70-80 \%$ of these patients $(1,2)$. Compared to people without diabetes, patients with T2DM are also more likely to have or develop more severe histological forms of NAFLD, such as nonalcoholic steatohepatitis (NASH), advanced fibrosis and cirrhosis, even in those patients with fairly "normal" serum liver enzyme levels (2-4). A recent meta-analysis has confirmed that the global prevalence of biopsy-confirmed NASH among individuals with T2DM was approximately $38 \%$ and that the global prevalence of advanced fibrosis in patients with NAFLD and T2DM was approximately $17 \%(2)$. It is also important to remember the clinical importance of bidirectional relationship between NAFLD and T2DM $(4,5)$. Notably, the coexistence of NAFLD and T2DM will worsen the course of both conditions. Coexisting T2DM not only increases risk of NAFLD progression to advanced fibrosis and cirrhosis, but also increases risk of incident hepatocellular carcinoma, liver-related hospital admissions and liverrelated deaths (4,6-9). Additionally, the presence of NAFLD makes achieving good glycemic control more difficult, exacerbates hepatic/peripheral insulin resistance and promotes atherogenic dyslipidemia, therefore, further increasing the risk of developing extra-hepatic diseases (such as chronic kidney disease [CKD] and cardiovascular complications), particularly in T2DM patients with advanced NAFLD $(3,4,10)$. Collectively, these findings support the conclusion that in patients with T2DM, diagnosis of, and treatment for, NAFLD should be considered a high clinical priority for diabetologists or endocrinologists caring for patients at risk of progressive NAFLD. It can, therefore, be argued that there is a case for systematic screening for NAFLD amongst patients at high risk of advanced NAFLD, such as those with T2DM. This conclusion is in accordance with the 2016 European (EASL-EASD-EASO) guidelines and the Italian (AISF) guidelines $(11,12)$, but not with the 2016 England and Wales National Institute for Care Excellence (NICE) NAFLD guidelines (13) or the 2018 American Association for the Study of Liver Diseases (AASLD) practice guidelines (14). The latter has recommended against a systematic screening for NAFLD in patients with T2DM, because of uncertainties surrounding diagnostic tests and treatment options, alongside a lack of knowledge related to the long- 
term benefits and the cost-effectiveness of screening (14). However, the AASLD guidelines have also recommended that there should be a high index of suspicion for NAFLD and NASH in patients with T2DM, and that clinical decision aids, such as vibrationcontrolled transient elastography, can be used to identify those at low or high risk for advanced liver fibrosis, i.e., the strongest predictor of long-term adverse clinical outcomes in NAFLD (14). Indeed, the measurement of liver stiffness (LSM) by vibrationcontrolled transient elastography, also known as FibroScan ${ }^{\circledR}$ apparatus, is emerging as a reliable diagnostic tool to stage non-invasively liver fibrosis in patients with various chronic liver diseases, including NAFLD (15).

Despite the high prevalence and serious clinical implications of NAFLD in T2DM patients, this condition is frequently overlooked in routine diabetes care $(2,4)$. Thus, increasing awareness about the importance of NAFLD in patients with T2DM among primary care physicians, specialists and health policy makers must be prioritized.

Therefore, the main aim of this cross-sectional study was to non-invasively screen the presence of NAFLD and liver fibrosis, by using both ultrasonography and vibrationcontrolled transient elastography, in a well-characterized sample of T2DM outpatients without known liver diseases, and to examine whether increased liver fibrosis is associated with presence of CKD and cardiovascular complications in these patients.

\section{MATERIALS AND METHODS}

\section{Patients}

In this study, we enrolled 137 (48.2\% men) patients with non-insulin-treated T2DM, who consecutively attended our diabetes outpatient service during a period of approximately 4 months, and who accepted to undergo liver ultrasonography and vibration-controlled transient elastography for diagnosing and staging NAFLD. We excluded all patients with: (a) history of significant alcohol consumption (defined as $>20$ grams of alcohol per day for men and women) and other known causes of chronic liver diseases (e.g., virus, drugs, autoimmunity and hemochromatosis); (b) history of cirrhosis of any etiology, active cancer, end-stage kidney disease (defined as estimated glomerular filtration rate [eGFR] 
$<15 \mathrm{ml} / \mathrm{min} / 1.73 \mathrm{~m}^{2}$ or dialysis) and impaired thyroid function; (c) treatment with insulin; and (d) chronic use of potentially hepatotoxic drugs, such as non-steroid antiinflammatory agents, steroids, tamoxifen, amiodarone or methotrexate, as well as treatment with hormone replacement therapy (only for women). According to the technical limitations of the vibration-controlled transient elastography, patients with congestive heart failure or free abdominal fluid were also excluded from the study.

The local Ethics Committee approved the study protocol. All patients gave their written informed consent for participation in this research.

\section{Clinical and laboratory data}

Body mass index (BMI) was measured as kilograms divided by the square of height in meters. Waist circumference was measured at the midpoint between the lowest rib and the iliac crest. Blood pressure was measured with a standard sphygmomanometer after the patient had been seated quietly for at least 5 minutes. Patients were considered to have hypertension if their blood pressure was $\geq 140 / 90 \mathrm{mmHg}$ or if they were taking any anti-hypertensive drugs.

Venous blood samples were collected in the morning after an overnight fast. Complete blood count, serum liver enzymes (aspartate aminotransferase [AST], alanine aminotransferase [ALT], gamma-glutamyltransferase [GGT]), lipids, creatinine (measured using a Jaffé rate blanked and compensated assay), high-sensitivity $C$ reactive protein (hsCRP) and other biochemical blood parameters were measured using standard laboratory procedures at the central Laboratory of our hospital, using the relative reference techniques on Roche Cobas 8000 (Roche Diagnostics, Basel, Switzerland). Low-density lipoprotein (LDL)-cholesterol was calculated using the Friedewald's equation. Dyslipidemia was defined as presence of plasma LDL cholesterol levels $>100 \mathrm{mg} / \mathrm{dL}$ (>2.5 $\mathrm{mmol} / \mathrm{L})$ or treatment with lipid-lowering drugs. Hemoglobin $\mathrm{A} 1 \mathrm{c}(\mathrm{HbA} 1 \mathrm{c})$ was measured using the high-performance liquid chromatography analyzer Tosoh-G7 (Tosoh Bioscience Inc., Tokyo, Japan). Fasting insulin concentrations were measured using a chemiluminescent immunoassay (LIAISON, DiaSorin, Saluggia, Italy). Homeostasis model assessment (HOMA-IR) score was used for estimating insulin resistance. 
Glomerular filtration rate was estimated using both the four-variable Modification of Diet in Renal Disease (MDRD) study equation (16), and the Chronic Kidney Disease Epidemiology Collaboration (CKD-EPI) equation (17). Urinary albumin excretion rate was assessed with an immuno-nephelometric assay (Beckman-Coulter IMMAGE; BeckmanCoulter Instruments, Fullerton, CA, USA) on a morning spot urine sample and expressed as the albumin-to-creatinine ratio (ACR); abnormal albuminuria was defined as a urinary $A C R \geq 30 \mathrm{mg} / \mathrm{g}$ creatinine. Chronic kidney disease (CKD) was defined as the presence of eGFR $<60 \mathrm{~mL} / \mathrm{min} / 1.73 \mathrm{~m}^{2}$ and/or abnormal albuminuria.

Presence of ischemic heart disease (IHD) was defined as a documented history of myocardial infarction, angina pectoris or coronary revascularization procedures. Presence of ischemic stroke was based on medical history and examination, and was confirmed by reviewing hospital medical records of patients, including radiology imaging results. The diagnosis of permanent atrial fibrillation was made based on standard 12-lead electrocardiograms and medical history (from reviewing hospital and physician charts from all patients). Presence of diabetic retinopathy, diagnosed with fundoscopy after pupillary dilation, was also recorded in all patients.

\section{Liver ultrasonography and liver stiffness measurement}

Hepatic steatosis was diagnosed by ultrasonography (using an Esaote MyLab 70 ultrasound with a $4 \mathrm{MHz}$ probe) according to specific ultrasonographic characteristics, such as diffuse hyper-echogenicity of the liver relative to kidneys, ultrasonographic beam attenuation, and poor visualization of intra-hepatic vessel borders and diaphragm. The ultrasonographic severity of hepatic steatosis was expressed with a semi-quantitative scale (i.e., mild, moderate or severe) (18).

Liver stiffness measurements (LSM) were performed using vibration-controlled transient elastography (Fibroscan ${ }^{\circledR}$ Echosens, Paris, France) with the M probe. Our Fibroscan ${ }^{\circledR}$ was not equipped with controlled attenuation parameter (CAP) for the assessment of hepatic steatosis. A trained physician performed LSMs in all patients after at least eight hours of fasting and after liver ultrasonography. Further details of the technical background and 
examination procedures have been described elsewhere (19). Briefly, each LSM measurement was considered adequate when including at least ten valid measurements for each patient, with a success rate $>60 \%$ and variability of measurements $<30 \%$ of the median $(15,19)$. The presence of clinically significant or advanced hepatic fibrosis was defined by the presence of either $L S M \geq 7 \mathrm{kPa}$ (that corresponds to Kleiner stage $\mathrm{F} \geq 2$ fibrosis on histology) or LSM $\geq 8.7 \mathrm{kPa}$ (corresponding to $\mathrm{F} \geq 3$ fibrosis on histology) $(15,20)$.

Both liver ultrasonography and vibration-controlled transient elastography were performed by a single expert physician, who was blinded to participants' clinical and biochemical details.

\section{Genetic analysis}

Genomic DNA was extracted from peripheral blood leukocytes using the QIAamp DNA Blood Mini Kit (Qiagen, Germany). Genotyping of both rs738409 in patatin-like phospholipase domain-containing protein-3 (PNPLA3) gene and rs58542926 in transmembrane 6 superfamily member 2 (TM6SF2) gene were carried out by a predesigned TaqMan probe (Applied Biosystem, California, USA), according to manufacturers' protocol. Polymorphism genotyping was performed using 7900 HT Real-Time PCR (Applied Biosystem, California, USA) (21).

\section{Statistical Analysis}

Continuous variables were expressed as means \pm SD or medians (inter-quartile range $[I Q R])$, categorical variables as proportions. The Fisher's exact test for categorical variables, the one-way analysis of variance for normally distributed continuous variables or the Kruskal-Wallis test for non-normally distributed variables (i.e., duration of diabetes, plasma triglycerides, fasting insulin, GGT levels, HOMA-IR score and LSM values) were used to examine the differences in clinical and biochemical characteristics among patients stratified by tertiles of LSM. The association between increased values of LSM (included either as $3^{\text {rd }}$ tertile vs. $1^{\text {st }}$ and $2^{\text {nd }}$ tertiles combined or as a continuous measure [for every SD increase in LSM, i.e. $2.4 \mathrm{kPa}$ ) and the presence of either CKD (i.e., eGFR <60 $\mathrm{mL} / \mathrm{min} / 1.73 \mathrm{~m}^{2}$ or abnormal albuminuria) or cardiovascular complications (i.e., prior IHD, ischemic stroke or permanent atrial fibrillation) was tested using both an unadjusted 
logistic regression model and three progressive multivariable logistic regression models with pre-specified adjustments for multiple established cardio-renal risk factors and potential confounders, such as age, sex, diabetes duration, HbA1c, smoking, hypertension, dyslipidemia, BMI, HOMA-IR score and plasma hs-CRP levels. All statistical tests were 2-sided and $p$-value of $<0.05$ (two-tailed) was considered statistically significant. Statistical analyses were performed using STATA software, version 14.2 (STATA, College Station, Texas, USA).

\section{RESULTS}

Overall, 137 patients with non-insulin-treated T2DM were in the study $(48.2 \%$ men; mean \pm SD: age $69.9 \pm 7$ years; $B M I 28.5 \pm 4.7 \mathrm{~kg} / \mathrm{m}^{2}$; waist circumference $100 \pm 12 \mathrm{~cm}$; HbA1c $6.9 \pm 0.8 \%$, median duration of diabetes 11 years [IQR 6-18]). Of these patients, 28 (20.4\%) had established cardiovascular disease (20 [14.6\%] patients had prior IHD and 8 [8.5\%] had either ischemic stroke or permanent atrial fibrillation). In addition, 37 (27\%) of these patients had CKD (14 [10.2\%] patients had eGFR $<60 \mathrm{ml} / \mathrm{min} / 1.73 \mathrm{~m}^{2}$ and 25 [18.2\%] had abnormal albuminuria). Most of these patients also had dyslipidemia (86.7\%) or hypertension (79.6\%).

As shown in Figure 1, in these 137 patients without known liver diseases the proportion of hepatic steatosis on ultrasonography was $73.7 \%$ (i.e., $41.6 \%$ had mild hepatic steatosis and $32.1 \%$ had moderate-to-severe steatosis), whereas the proportion with significant/advanced liver fibrosis was $17.5 \%$ when using a LSM cutoff value $\geq 7 \mathrm{kPa}$ or $10.2 \%$ when using a LSM cutoff value $\geq 8.7 \mathrm{kPa}$.

Table 1 shows the clinical and biochemical characteristics of patients, stratified by LSM tertiles. Compared to patients in the $1^{\text {st }}$ tertile who had a median LSM of $3.6 \mathrm{kPa}$ [IQR 3.3.-4.0 kPa], patients with increased liver fibrosis (i.e., those belonging to the $3^{\text {rd }}$ tertile of LSM who had a median of 7.8 kPa [IQR 6.8-9.5 kPa]) were more likely to be centrally obese and had greater insulin resistance, higher plasma triglycerides, higher serum liver enzyme levels (although they were largely within the normal laboratory range in most of 
our patients) and greater proportion of hepatic steatosis on ultrasonography. Age, sex, duration of diabetes, smoking, hypertension, dyslipidemia, diabetic retinopathy, as well as white blood cell count, platelet count and circulating levels of $\mathrm{HbA} 1 \mathrm{c}$, creatinine and hs-CRP were not significantly different among the three patient groups. Moreover, there were no significant differences in the distribution of PNPLA3 and TM6SF2 genetic variants among the three groups.

As reported in Table 2, no significant differences were also found in the use of antihyperglycemic agents (by study design no patients were treated with insulin), antihypertensive, antiplatelet or lipid-lowering medications among the three groups of patients.

Figure 2 shows that the proportion of CKD (eGFR $<60 \mathrm{~mL} / \mathrm{min} / 1.73 \mathrm{~m}^{2}$ or abnormal albuminuria) increased progressively across LSM tertiles, from approximately $15 \%$ in the $1^{\text {st }}$ tertile to $45 \%$ in $3^{\text {rd }}$ tertile of LSM ( $p<0.01$ by the Fisher's exact test). Cardiovascular complications also tended to increase across LSM tertiles (from approximately $15 \%$ to $30 \%$ in $1^{\text {st }} v s .3^{\text {rd }}$ tertile) but did not achieve statistical significance.

After stratifying the patients by severity of hepatic steatosis on ultrasonography, we failed to find any significant difference in the proportion of CKD (27.9\% vs. $17.5 \%$ vs. $38.8 \% ; p=0.10$ by the Fisher's exact test) and cardiovascular complications ( $18.2 \%$ vs. $21.1 \%$ vs. $22.2 \% ; p=0.84$ ) among patients with moderate-severe steatosis vs. mild steatosis vs. no steatosis, respectively.

Table 3 shows the association between increased liver fibrosis ( $3^{\text {rd }}$ tertile of LSM vs. $1^{\text {st }}$ and $2^{\text {nd }}$ tertiles combined) and presence of either CKD or cardiovascular complications as well as the effect of adjustment for multiple established risk factors and potential confounders on these associations. In univariable logistic regression analysis (unadjusted model), increased liver fibrosis was associated with a nearly three-fold higher risk of prevalent CKD (unadjusted odds ratio [OR] 3.03, 95\% $\mathrm{Cl} 1.36-6.78, p=0.007$ ), as well as with an approximately 2.2-fold increased risk of cardiovascular complications (unadjusted OR $2.17,95 \% \mathrm{Cl} 0.93-5.14, p=0.069)$. Adjustment for established cardio-renal risk factors, 
such as age, sex, duration of diabetes, HbA1c, smoking, hypertension and dyslipidemia (adjusted model 2) did not essentially modify the strong association between increased liver fibrosis and risk of CKD (adjusted-OR 3.53, 95\% Cl 1.46-8.52, $p=0.005$ ), whereas the association between increased liver fibrosis and risk of cardiovascular complications became statistically significant (adjusted-OR 2.85, 95\% Cl 1.07-7.59, $p=0.036$ ). However, after further adjustment for BMI, HOMA-IR score and plasma hs-CRP levels (adjusted model 3), increased liver fibrosis retained a significant association only with the risk of CKD (adjusted-OR 3.28, 95\% Cl 1.22-8.90, $p=0.019$ ), but not with that of cardiovascular complications. Other variables that were independently associated with increased risk of prevalent CKD were male sex (adjusted-OR 3.15, 95\% Cl 1.27-7.84, $p=0.005$ ) and hypertension (adjusted-OR 3.56, 95\% Cl 1.02-12.9, $p=0.005$ ).

Regarding the association between increased liver fibrosis and risk of CKD, almost identical results were found even when the CKD-EPI formula was used for calculating eGFR instead of the MDRD study formula (data not shown).

Supplementary Table 1 shows the association between increasing LSM values (included as a continuous measure, i.e., for each SD increment that corresponds to $2.4 \mathrm{kPa}$ ) and the presence of either CKD or cardiovascular complications as well as the effect of adjustment for established risk factors and potential confounders on these associations. Both in univariable logistic regression analyses and in multivariable logistic regression analyses (adjusted model 3), increasing values of LSM were significantly associated with an increased risk of both prevalent CKD (adjusted-OR 1.68, 95\% $\mathrm{Cl} 1.04-2.70, p=0.034$ ) and cardiovascular complications (adjusted-OR 1.73, 95\% Cl 1.04-2.88, $p=0.035$ ).

\section{DISCUSSION}

A major finding of our cross-sectional study is that a substantial proportion of patients with T2DM attending a routine outpatient service had NAFLD and significant/advanced liver fibrosis. The majority of these patients had hepatic steatosis on ultrasonography (i.e. 
75\%), and a significant proportion of them also had increased liver fibrosis $(17.5 \%$ had LSM $\geq 7 \mathrm{kPa}$ and $10.2 \%$ had $\mathrm{LSM} \geq 8.7 \mathrm{kPa}$ on vibration-controlled transient elastography, corresponding to Kleiner stage $F \geq 3$ fibrosis on histology) $(14,19)$. It is also noteworthy that the vast majority of our patients with T2DM had fairly normal serum liver enzymes, thus emphasising that serum liver enzyme levels cannot be regarded as reliable markers for the screening and diagnosis of NAFLD in patients with T2DM, and should not be used to this end in clinical practice $(4,11,13,14)$.

Our findings are in line with recent results of a few studies that used vibration-controlled transient elastography $\left(\right.$ FibroScan $\left.^{\circledR}\right)$ or other non-invasive imaging techniques for staging liver fibrosis in patients with T2DM. A small study using magnetic resonance imaging for assessing NAFLD and magnetic resonance elastography for measuring liver stiffness has reported a high proportion of hepatic steatosis and advanced fibrosis (65\% and $7 \%$, respectively) in an outpatient sample of 100 United States obese T2DM individuals without known liver diseases (22). Kwok et al. also reported a high proportion of hepatic steatosis ( $73 \%)$ and advanced fibrosis ( $17.7 \%$ subjects had $L S M \geq 9.6 \mathrm{kPa}$ ) in a hospitalbased cohort of nearly 1,800 Chinese patients with T2DM (54\% male; mean age 60.6 years; mean BMI $26.6 \mathrm{~kg} / \mathrm{m}^{2}$; mean $\mathrm{HbA1c} 7.4 \%$ ), where liver fat and fibrosis were assessed with FibroScan ${ }^{\circledR}(23)$. Notably, in a subset of these patients who underwent liver biopsy, $56 \%$ had NASH, $21 \%$ had advanced fibrosis and $29 \%$ had cirrhosis (23). Using the FibroScan ${ }^{\circledR}$, Roulot et al. found that the proportion of advanced fibrosis was $12.7 \%$ (defined as LSM $\geq 8 \mathrm{kPa}$ ) in a cohort of $\sim 700$ French patients with T2DM (56\% male; mean age 58 years; mean BMI $29.6 \mathrm{~kg} / \mathrm{m}^{2}$; HbA1c 7.7\%) (24). Recently, in 557 Malaysian patients with T2DM (41\% male; mean age 61 years; mean BMI $28.2 \mathrm{~kg} / \mathrm{m}^{2}$; mean HbA1c 7.7\%) attending the diabetes clinic of an university hospital, Lai et al. reported that the proportion of NAFLD and advanced fibrosis (defined as LSM $\geq 8 \mathrm{kPa}$ ), based on FibroScan ${ }^{\circledR}$, were $72.4 \%$ and $21 \%$, respectively $(25)$.

Our work extends previous findings as another important result of our study is that increased liver fibrosis (i.e., $3^{\text {rd }}$ tertile of LSM vs. $1^{\text {st }}+2^{\text {nd }}$ tertiles combined) is significantly associated with a $\sim 3$-fold higher risk of prevalent CKD $(p=0.007)$ and with a borderline significant $\sim 2.2$-fold increased risk of cardiovascular complications $(p=0.069)$. After 
adjustment for established risk factors, diabetes-related variables and other potential confounders, we found that only the association between increased LSM and risk of CKD maintained statistical significance, whereas the association between increased LSM and risk of cardiovascular complications was no longer significant. However, when LSM was included as a continuous measure in the aforementioned multivariable logistic regression models, we found that there was a significant and independent association between increasing LSM values and risk of both complications.

Previous studies have explored the association between imaging-defined NAFLD and the presence of CKD or clinically manifest cardiovascular disease in community-based or hospital-based cohorts of patients with T2DM (as extensively reviewed in $[4,26,27]$ ). For example, Targher et al. firstly reported that NAFLD on ultrasonography was significantly associated with a higher prevalence of cardiovascular disease (28), as well as with higher prevalence of CKD and proliferative/laser-treated retinopathy (29), independent of traditional cardiovascular risk factors, glycemic control, medication use and metabolic syndrome features. To our knowledge, however, the usefulness of vibration-controlled transient elastography in the risk prediction of CKD has been explored only in very few studies involving patients with T2DM. Recently, Yeung et al. found that advanced liver fibrosis (defined as LSM $\geq 9.6 \mathrm{kPa}$ ), but not steatosis, was independently associated with abnormal albuminuria in a hospital cohort of nearly 1,800 Chinese patients with T2DM (30). At present, no studies are available in the literature examining the usefulness of vibration-controlled transient elastography (FibroScan ${ }^{\circledR}$ ) in risk prediction of clinically manifest cardiovascular complications in patients with T2DM.

Collectively, the findings of our study are of potential clinical importance for management of patients with T2DM, who rarely undergo liver biopsy or other imaging techniques for diagnosing and staging NAFLD in routine diabetes care. Indeed, the present results show that there is a high proportion of NAFLD and significant/advanced liver fibrosis among our metabolically well-controlled patients with non-insulin-treated T2DM without known liver diseases (most of whom had fairly normal serum liver enzyme levels). These data also support the assertion that vibration-controlled transient elastography (FibroScan ${ }^{\circledR}$ ) can be $^{2}$ useful not only for assessing the severity of liver fibrosis, which is now regarded as the 
strongest predictor of long-term adverse clinical outcomes in $\operatorname{NAFLD}(3,11,14,20)$ but also for identifying patients at higher risk of having CKD or other chronic vascular complications. In addition, due to the fact that there were no significant differences in the use of any anti-hyperglycemic agents across LSM tertiles among our T2DM patients, these findings also suggest that more widespread use of vibration-controlled transient elastography (FibroScan ${ }^{\circledR}$ ) in patients with T2DM could also allow a better choice of the pharmacological treatment for diabetes using some anti-hyperglycemic agents (if not contraindicated), which may have significant benefits on NASH and liver fibrosis, such as pioglitazone and liraglutide (11-14,31-34). All these findings highlight the importance of increasing awareness of the prognostic value of NAFLD in patients with T2DM among primary care physicians and diabetologists/endocrinologists. An accurate, patientcentred, team-based approach to the management and treatment of individuals with T2DM and NAFLD, based on a careful evaluation of related cardio-metabolic risk factors and monitoring for cardiovascular, renal and liver complications, is warranted $(4,35)$.

Our study has some important limitations that should be mentioned. First, the crosssectional design of this single-centre study limits our ability to establish causal or temporal relationships between increased liver fibrosis and risk of CKD and cardiovascular complications. Second, the patient cohort comprised exclusively Caucasian individuals with metabolically well-controlled, non-insulin-treated T2DM without known liver diseases, attending a diabetes outpatient service. Thus, results cannot be necessarily extrapolated to the general population of patients with T2DM or other ethnic groups. On the other hand, this limitation represents a specific strength by showing that the proportion of hepatic steatosis and increased liver fibrosis are high in the absence of major changes in glycemia. Third, we did not perform liver biopsies, so we are not able to compare the results of liver stiffness obtained by FibroScan ${ }^{\circledR}$ with histology data. That said, vibration-controlled transient elastography (FibroScan ${ }^{\circledR}$ ) is a reliable non-invasive method for staging liver fibrosis in $\operatorname{NAFLD}(15,36)$. Finally, due to the relatively small number of patients with CKD or prior cardiovascular diseases, the interpretation of results of our fully adjusted logistic regression models requires some caution. Further studies in larger cohorts of patients are needed to further examine this issue. 
Despite these limitations, our study has some important strengths, including the consecutive enrollment of the study population, the completeness of the database, and the adjustment for multiple established cardio-renal risk factors, diabetes-related variables and potential confounders. In addition, measurements of LSM were performed (in all patients) by a single trained physician, thus eliminating potential inter-observer reproducibility.

In conclusion, our results suggest that NAFLD and increased liver fibrosis (on vibrationcontrolled transient elastography) were very common in a well-characterized sample of outpatients with non-insulin-treated T2DM without known liver diseases, and that increased liver fibrosis was strongly associated with a higher prevalence of chronic vascular complications of diabetes, especially CKD. These results highlight the need to screen patients with T2DM for CKD and cardiovascular complications when increased liver fibrosis is detected. Similarly, liver fibrosis should be assessed in patients with T2DM when CKD or cardiovascular disease is detected.

Disclosure Statement: The Authors have no potential conflicts of interest to disclose.

Funding: GT is supported in part by grants from the University School of Medicine of Verona, Verona, Italy. CDB is supported in part by grants from the Southampton National Institute for Health Research Biomedical Research Centre.

\section{REFERENCES}

1. Lonardo A, Bellentani S, Argo CK, Ballestri S, Byrne CD, Caldwell SH, Cortez-Pinto H, Grieco A, Machado MV, Miele L, Targher G. Epidemiological modifiers of nonalcoholic fatty liver disease: focus on high-risk groups. Dig Liver Dis. 2015;47:9971006.

2. Younossi ZM, Golabi P, de Avila L, Paik JM, Srishord M, Fukui N, Qiu Y, Burns L, Afendy A, Nader F. The global epidemiology of NAFLD and NASH in patients with type 2 diabetes: A systematic review and meta-analysis. J Hepatol. 2019 Jul 4. pii: S01688278(19)30393-9. doi: 10.1016/j.jhep.2019.06.021 [Epub ahead of print]. 
3. Anstee QM, Targher G, Day CP. Progression of NAFLD to diabetes mellitus, cardiovascular disease or cirrhosis. Nat Rev Gastroenterol Hepatol. 2013;10:330-344.

4. Targher G, Lonardo A, Byrne CD. Nonalcoholic fatty liver disease and chronic vascular complications of diabetes mellitus. Nat Rev Endocrinol. 2018;14:99-114.

5. Targher G, Marchesini G, Byrne CD. Risk of type 2 diabetes in patients with nonalcoholic fatty liver disease: causal association or epiphenomenon? Diabetes Metab. 2016;42:142-156.

6. Porepa L, Ray JG, Sanchez-Romeu P, Booth GL. Newly diagnosed diabetes mellitus as a risk factor for serious liver disease. CMAJ. 2010;182:E526-E531.

7. Wild SH, Morling JR, McAllister DA, Kerssens J, Fischbacher C, Parkes J, Roderick PJ, Sattar N, Byrne CD; Scottish and Southampton Diabetes and Liver Disease Group; Scottish Diabetes Research Network Epidemiology Group. Type 2 diabetes and risk of hospital admission or death for chronic liver diseases. J Hepatol. 2016;64:1358-1364.

8. Zoppini G, Fedeli U, Gennaro N, Saugo M, Targher G, Bonora E. Mortality from chronic liver diseases in diabetes. Am J Gastroenterol. 2014;109:1020-1025.

9. Singal AG, El-Serag HB. Hepatocellular carcinoma from epidemiology to prevention: translating knowledge into practice. Clin Gastroenterol Hepatol. 2015;13:2140-2151.

10. Targher G, Byrne CD, Lonardo A, Zoppini G, Barbui C. Non-alcoholic fatty liver disease and risk of incident cardiovascular disease: A meta-analysis. J Hepatol. 2016;65:589600.

11. European Association for the Study of the Liver (EASL); European Association for the Study of Diabetes (EASD); European Association for the Study of Obesity (EASO). EASL-EASD-EASO Clinical Practice Guidelines for the management of non-alcoholic fatty liver disease. J Hepatol. 2016;64:1388-1402.

12. Italian Association for the Study of the Liver (AISF). AISF position paper on nonalcoholic fatty liver disease (NAFLD): updates and future directions. Dig Liver Dis 2017;49:471-483.

13. Glen J, Floros L, Day C, Pryke R; Guideline Development Group. Non-alcoholic fatty liver disease (NAFLD): summary of NICE guidance. BMJ. 2016;354:i4428.

14. Chalasani N, Younossi Z, Lavine JE, Charlton M, Cusi K, Rinella M, Harrison SA, Brunt EM, Sanyal AJ. The diagnosis and management of nonalcoholic fatty liver disease: Practice guidance from the American Association for the Study of Liver Diseases. Hepatology. 2018;67:328-357.

15. Castera L, Friedrich-Rust M, Loomba R. Noninvasive assessment of liver disease in patients with nonalcoholic fatty liver disease. Gastroenterology. 2019;156:12641281.e4.

16. Levey AS, Bosch JP, Lewis JB, Greene T, Rogers N, Roth D. A more accurate method to estimate glomerular filtration rate from serum creatinine: a new prediction equation. Modification of Diet in Renal Disease Study Group. Ann Intern Med. 1999;130:461470.

17. Levey AS, Stevens LA, Schmid CH, Zhang YL, Castro AF 3rd, Feldman HI, Kusek JW, Eggers P, Van Lente F, Greene T, Coresh J; CKD-EPI (Chronic Kidney Disease Epidemiology Collaboration). A new equation to estimate glomerular filtration rate. Ann Intern Med. 2009;150:604-612.

18. Mantovani A, Sani E, Fassio A, Colecchia A, Viapiana O, Gatti D, Idolazzi L, Rossini M, Salvagno G, Lippi G, Zoppini G, Byrne CD, Bonora E, Targher G. Association between non-alcoholic fatty liver disease and bone turnover biomarkers in post-menopausal women with type 2 diabetes. Diabetes Metab. 2019;45:347-355. 
19. Mikolasevic I, Orlic L, Franjic N, Hauser G, Stimac D, Milic S. Transient elastography (FibroScan ${ }^{\circledR}$ ) with controlled attenuation parameter in the assessment of liver steatosis and fibrosis in patients with nonalcoholic fatty liver disease - Where do we stand? World J Gastroenterol. 2016;22:7236-72351.

20. Byrne CD, Patel J, Scorletti E, Targher G. Tests for diagnosing and monitoring nonalcoholic fatty liver disease in adults. BMJ. 2018;362:k2734.

21. Mantovani A, Zusi C, Sani E, Colecchia A, Lippi G, Zaza GL, Valenti L, Byrne CD, Maffeis C, Bonora E, Targher G. Association between PNPLA3rs738409 polymorphism decreased kidney function in postmenopausal type 2 diabetic women with or without non-alcoholic fatty liver disease. Diabetes Metab. 2019 Feb 11. pii: S12623636(19)30026-6. doi: 10.1016/j.diabet.2019.01.011 [Epub ahead of print].

22. Doycheva I, Cui J, Nguyen P, Costa EA, Hooker J, Hofflich H, Bettencourt R, Brouha S, Sirlin CB, Loomba R. Non-invasive screening of diabetics in primary care for NAFLD and advanced fibrosis by MRI and MRE. Aliment Pharmacol Ther. 2016;43:83-95.

23. Kwok R, Choi KC, Wong GL, Zhang Y, Chan HL, Luk AO, Shu SS, Chan AW, Yeung MW, Chan JC, Kong AP, Wong VW. Screening diabetic patients for non-alcoholic fatty liver disease with controlled attenuation parameter and liver stiffness measurements: a prospective cohort study. Gut. 2016;65:1359-1368.

24. Roulot D, Roudot-Thoraval F, NKontchou G, Kouacou N, Costes JL, Elourimi G, Le Clesiau $\mathrm{H}$, Ziol M, Beaugrand $\mathrm{M}$. Concomitant screening for liver fibrosis and steatosis in French type 2 diabetic patients using Fibroscan. Liver Int. 2017;37:1897-1906.

25. Lai LL, Wan Yusoff WNI, Vethakkan SR, Nik Mustapha NR, Mahadeva S, Chan WK. Screening for non-alcoholic fatty liver disease in patients with type 2 diabetes mellitus using transient elastography. J Gastroenterol Hepatol. 2019;34:1396-1403.

26. Targher G, Byrne CD. Non-alcoholic fatty liver disease: an emerging driving force in chronic kidney disease. Nat Rev Nephrol. 2017;13:297-310.

27. Musso G, Gambino R, Tabibian JH, Ekstedt M, Kechagias S, Hamaguchi M, Hultcrantz R, Hagström H, Yoon SK, Charatcharoenwitthaya P, George J, Barrera F, Hafliđadóttir S, Björnsson ES, Armstrong MJ, Hopkins L, Gao X, Francque S, Verrijken A, Yilmaz Y, Lindor KD, Charlton M, Haring R, Lerch MM, Rettig R, Völzke H, Ryu S, Li G, Wong LL, Machado M, Cortez-Pinto H, Yasui K, Cassader M. Association of non-alcoholic fatty liver disease with chronic kidney disease: a systematic review and meta-analysis. PLoS Med 2014;11:e1001680.

28. Targher G, Bertolini L, Padovani R, Rodella S, Tessari R, Zenari L, Day C, Arcaro G. Prevalence of nonalcoholic fatty liver disease and its association with cardiovascular disease among type 2 diabetic patients. Diabetes Care. 2007;30:1212-1218.

29. Targher G, Bertolini L, Rodella S, Zoppini G, Lippi G, Day C, Muggeo M. Non-alcoholic fatty liver disease is independently associated with an increased prevalence of chronic kidney disease and proliferative/laser-treated retinopathy in type 2 diabetic patients. Diabetologia. 2008;51:444-450.

30. Yeung MW, Wong GL, Choi KC, Luk AO, Kwok R, Shu SS, Chan AW, Lau ESH, Ma RCW, Chan HL, Chan JC, Wong VW, Kong AP. Advanced liver fibrosis but not steatosis is independently associated with albuminuria in Chinese patients with type 2 diabetes. J Hepatol. 2018;68:147-156.

31. Cusi K, Orsak B, Bril F, Lomonaco R, Hecht J, Ortiz-Lopez C, Tio F, Hardies J, Darland C, Musi N, Webb A, Portillo-Sanchez P. Long-term pioglitazone treatment for patients with nonalcoholic steatohepatitis and prediabetes or type 2 diabetes mellitus: a randomized trial. Ann Intern Med. 2016;165:305-315. 
32. Musso G, Cassader M, Paschetta E, Gambino R. Thiazolidinediones and advanced liver fibrosis in nonalcoholic steatohepatitis: a meta-analysis. JAMA Intern Med. 2017;177:633-640.

33. Armstrong MJ, Gaunt P, Aithal GP, Barton D, Hull D, Parker R, Hazlehurst JM, Guo K; LEAN trial team, Abouda G, Aldersley MA, Stocken D, Gough SC, Tomlinson JW, Brown RM, Hübscher SG, Newsome PN. Liraglutide safety and efficacy in patients with non-alcoholic steatohepatitis (LEAN): a multicentre, double-blind, randomised, placebo-controlled phase 2 study. Lancet. 2016;387:679-690.

34. Amstrong MJ, Houlihan DD, Rowe IA, Clausen WH, Elbrønd B, Gough SC, Tomlinson JW, Newsome PN. Safety and efficacy of liraglutide in patients with type 2 diabetes and elevated liver enzymes: individual patient data meta-analysis of the LEAD program. Aliment Pharmacol Ther. 2013;37:234-242.

35. Anstee QM, Mantovani A, Tilg H, Targher G. Risk of cardiomyopathy and cardiac arrhythmias in patients with nonalcoholic fatty liver disease. Nat Rev Gastroenterol Hepatol. 2018;15:425-439.

36. Younossi ZM, Loomba R, Anstee QM, Rinella ME, Bugianesi E, Marchesini G, Neuschwander-Tetri BA, Serfaty L, Negro F, Caldwell SH, Ratziu V, Corey KE, Friedman SL, Abdelmalek MF, Harrison SA, Sanyal AJ, Lavine JE, Mathurin P, Charlton MR, Goodman ZD, Chalasani NP, Kowdley KV, George J, Lindor K. Diagnostic modalities for nonalcoholic fatty liver disease, nonalcoholic steatohepatitis, and associated fibrosis. Hepatology. 2018;68:349-360. 
Table 1. Clinical and biochemical characteristics of patients with non-insulin-treated T2DM stratified by tertiles of liver stiffness measurement (LSM assessed by vibration-controlled transient elastography).

\begin{tabular}{|c|c|c|c|c|}
\hline & $\begin{array}{l}\text { LSM } \\
\text { 1st tertile ( } n=49)\end{array}$ & $\begin{array}{l}\text { LSM } \\
\text { 2nd tertile ( } n=48)\end{array}$ & $\begin{array}{l}\text { LSM } \\
\text { 3rd tertile }(n=40)\end{array}$ & $P$-value \\
\hline Sex (\% female) & 59.2 & 47.9 & 47.5 & 0.442 \\
\hline Age (years) & $71.5 \pm 7$ & $69.3 \pm 7$ & $68.5 \pm 8$ & 0.129 \\
\hline Weight (kg) & $74 \pm 15$ & $77 \pm 13$ & $84 \pm 14$ & 0.005 \\
\hline BMI $\left(\mathrm{kg} / \mathrm{m}^{2}\right)$ & $27.9 \pm 5.6$ & $27.7 \pm 3.8$ & $30.2 \pm 3.8$ & 0.022 \\
\hline Waist circumference $(\mathrm{cm})$ & $96.7 \pm 13$ & $99.1 \pm 13$ & $105.0 \pm 10$ & 0.005 \\
\hline Diabetes duration (years) & $10(7-19)$ & $11(5-18)$ & $10(7-16)$ & 0.768 \\
\hline Current smokers (\%) & 4.1 & 18.8 & 12.5 & 0.230 \\
\hline Family history for cardiovascular disease (\%) & 31.1 & 40.6 & 37.5 & 0.671 \\
\hline Family history for diabetes (\%) & 59.1 & 60.0 & 70.3 & 0.544 \\
\hline Systolic blood pressure (mmHg) & $136 \pm 16$ & $131 \pm 16$ & $135 \pm 18$ & 0.306 \\
\hline Diastolic blood pressure (mmHg) & $76 \pm 9$ & $75 \pm 9$ & $77 \pm 9$ & 0.642 \\
\hline Hypertension (\%) & 79.5 & 81.2 & 77.5 & 0.964 \\
\hline Dyslipidemia (\%) & 93.9 & 83.3 & 82.5 & 0.188 \\
\hline Fasting glucose $(\mathrm{mg} / \mathrm{dL})$ & $129 \pm 31$ & $124 \pm 24$ & $133 \pm 29$ & 0.395 \\
\hline Hemoglobin A1c (\%) & $6.9 \pm 0.7$ & $6.8 \pm 0.6$ & $7.0 \pm 0.8$ & 0.481 \\
\hline Hemoglobin A1c $\geq 8 \%(\%)$ & 8.2 & 6.2 & 10.0 & 0.923 \\
\hline Total cholesterol (mg/dL) & $157 \pm 37$ & $153 \pm 37$ & $156 \pm 33$ & 0.843 \\
\hline LDL cholesterol $(\mathrm{mg} / \mathrm{dL})$ & $82 \pm 34$ & $77 \pm 31$ & $74 \pm 27$ & 0.455 \\
\hline $\mathrm{HDL}$ cholesterol $(\mathrm{mg} / \mathrm{dL})$ & $54.2 \pm 12$ & $52 \pm 14$ & $54 \pm 19$ & 0.610 \\
\hline Triglycerides (mg/dL) & 99 (71-135) & $110(84-161)$ & $132(97-186)$ & 0.005 \\
\hline Fasting insulin (mU/L) & $5.4(3.4-8.6)$ & $6.3(3.1-8.9)$ & $10.0(5.1-19.9)$ & $<0.001$ \\
\hline HOMA-IR score & $1.8(1.0-2.6)$ & $1.8(1.0-2.5)$ & $3.4(2.0-6.1)$ & $<0.001$ \\
\hline Creatinine (umol/L) & $73.3 \pm 19$ & $72.8 \pm 20$ & $78.2 \pm 22$ & 0.402 \\
\hline Hemoglobin $(\mathrm{g} / \mathrm{L})$ & $134 \pm 15$ & $139 \pm 11$ & $140 \pm 12$ & 0.094 \\
\hline White blood count $\left(\times 10^{9} / L\right)$ & $7.12 \pm 1.6$ & $7.40 \pm 2.0$ & $7.21 \pm 1.5$ & 0.737 \\
\hline Platelet count $\left(\times 10^{9} / \mathrm{L}\right)$ & $243 \pm 59$ & $240 \pm 58$ & $234 \pm 62$ & 0.797 \\
\hline AST (IU/L) & $23 \pm 8$ & $23 \pm 5$ & $27 \pm 10$ & 0.021 \\
\hline ALT (IU/L) & $14 \pm 8$ & $13 \pm 6$ & $17 \pm 9$ & 0.058 \\
\hline GGT (IU/L) & $15(10-20)$ & $21(14-30)$ & $27(17-40)$ & $<0.001$ \\
\hline hs-CRP (mg/L) & $1.01(0.5-2.9)$ & $0.98(0.4-2.7)$ & $2.05(0.7-3.9)$ & 0.976 \\
\hline Diabetic retinopathy, any degree (\%) & 8.5 & 8.3 & 12.8 & 0.868 \\
\hline Hepatic steatosis on ultrasonography (\%) & 59.2 & 70.8 & 95.0 & $<0.001$ \\
\hline PNPLA3 rs738409 & & & & 0.672 \\
\hline CC genotype (\%) & 46.9 & 47.9 & 52.5 & \\
\hline CG genotype (\%) & 48.9 & 47.9 & 37.5 & \\
\hline GG genotype (\%) & 4.2 & 4.2 & 10.0 & \\
\hline TM6SF2 rs58542926 & & & & 0.945 \\
\hline CC genotype (\%) & 85.7 & 89.6 & 87.5 & \\
\hline CT genotype (\%) & 14.3 & 10.4 & 12.5 & \\
\hline TT genotype (\%) & 0 & 0 & 0 & \\
\hline LSM by FibroScan ${ }^{\circledast}(\mathrm{kPa})$ & $3.6(3.3-4.0)$ & $5.3(4.9-5.7)$ & $7.8(6.8-9.5)$ & ND \\
\hline
\end{tabular}

Sample size, $n=137$. Data are expressed as means \pm SD, medians and IQR (in parenthesis) or proportions. Differences among the three patient groups were tested by the one-way ANOVA for continuous variables normally distributed, the Kruskal-Wallis test for variables not normally distributed and the Fisher exact test for categorical variables. For the sake of clarity significant $p$-values are highlighted in bold. Hypertension was defined as blood pressure $\geq 140 / 90$ mmHg or use of any anti-hypertensive drugs. Dyslipidemia was defined as plasma LDL-cholesterol levels $>100 \mathrm{mg} / \mathrm{dL}$ or use of lipid-lowering agents.

Abbreviations: ALT, alanine aminotransferase; AST, aspartate aminotransferase; BMI, body mass index; GGT, gamma-glutamyltransferase; hs-CRP, high sensitivity C-reactive protein; HOMA-IR, homeostasis model assessment-insulin resistance; LSM, liver stiffness measurement; ND, not determined; PNPLA3, patatin-like phospholipase domain-containing protein 3; TM6SF2, trans-membrane 6 superfamily member 2. 
Table 2. Drug treatments in patients with non-insulin-treated T2DM stratified by tertiles of liver stiffness measurement (LSM assessed by vibration-controlled transient elastography).

\begin{tabular}{|c|c|c|c|c|}
\hline & $\begin{array}{l}\text { LSM } \\
\text { 1st tertile }(n=49)\end{array}$ & $\begin{array}{l}\text { LSM } \\
\text { 2nd tertile }(n=48)\end{array}$ & $\begin{array}{l}\text { LSM } \\
\text { 3rd tertile }(n=40)\end{array}$ & $P$-value \\
\hline Metformin (\%) & 83.7 & 83.3 & 92.5 & 0.408 \\
\hline Sulphonylureas (\%) & 24.5 & 25.0 & 30.0 & 0.857 \\
\hline DPP-4 inhibitors (\%) & 26.5 & 25.0 & 30.0 & 0.860 \\
\hline GLP-1 agonists (\%) & 16.3 & 22.9 & 15.0 & 0.573 \\
\hline SGLT-2 inhibitors (\%) & 6.1 & 10.4 & 12.5 & 0.583 \\
\hline Pioglitazone (\%) & 2.0 & 10.4 & 10.0 & 0.184 \\
\hline Acarbose (\%) & 6.1 & 2.1 & 5.0 & 0.674 \\
\hline ARB/ACE-inhibitors (\%) & 60.4 & 63.8 & 65.0 & 0.925 \\
\hline Calcium-channel blockers (\%) & 16.7 & 21.3 & 27.5 & 0.455 \\
\hline Beta-blockers (\%) & 37.5 & 25.5 & 37.5 & 0.373 \\
\hline Diuretics (\%) & 35.4 & 25.5 & 32.5 & 0.569 \\
\hline Statins (\%) & 83.7 & 80.9 & 77.5 & 0.764 \\
\hline Fibrates (\%) & 2.1 & 0.0 & 7.5 & 0.106 \\
\hline Antiplatelets (\%) & 56.2 & 40.4 & 52.5 & 0.275 \\
\hline
\end{tabular}

Sample size, $n=137$. Data are expressed as proportions. Differences among the three groups of patients were tested by the Fisher's exact test.

Abbreviations: ACE, angiotensin-converting-enzyme; ARB, angiotensin II receptor blocker; DPP-4, dipeptidyl peptidase-4; GLP-1, glucagon-like peptide-1; SGLT-2, sodium/glucose cotransporter-2; LSM, liver stiffness measurement. 
Table 3. Association between increased liver stiffness measurement on vibration-controlled transient elastography (included as categorical measure) and the presence of either chronic kidney disease or cardiovascular complications in patients with non-insulin-treated T2DM.

\begin{tabular}{|c|c|c|c|c|}
\hline & \multicolumn{2}{|c|}{ Chronic kidney disease } & \multicolumn{2}{|c|}{ Cardiovascular complications } \\
\hline & Odds ratio $(95 \% \mathrm{Cl})$ & $P$-value & Odds ratio $(95 \% \mathrm{Cl})$ & $P$-value \\
\hline \multicolumn{5}{|l|}{ Unadjusted model } \\
\hline LSM $\left(3^{\circ}\right.$ vs. $1^{\circ}+2^{\circ}$ tertiles combined) & $3.03(1.36-6.78)$ & 0.007 & $2.17(0.93-5.14)$ & 0.069 \\
\hline \multicolumn{5}{|l|}{ Adjusted model 1} \\
\hline LSM $\left(3^{\circ}\right.$ vs. $1^{\circ}+2^{\circ}$ tertiles combined $)$ & $3.01(1.32-6.87)$ & 0.007 & $2.21(0.94-5.34)$ & 0.068 \\
\hline \multicolumn{5}{|l|}{ Adjusted model 2} \\
\hline LSM $\left(3^{\circ}\right.$ vs. $1^{\circ}+2^{\circ}$ tertiles combined) & $3.53(1.46-8.52)$ & 0.005 & 2.85 (1.07-7.59) & 0.036 \\
\hline \multicolumn{5}{|l|}{ Adjusted model 3} \\
\hline $\operatorname{LSM}\left(3^{\circ}\right.$ vs. $1^{\circ}+2^{\circ}$ tertiles combined) & $3.28(1.22-8.90)$ & 0.019 & $2.39(0.80-7.45)$ & 0.125 \\
\hline
\end{tabular}

Data are expressed as odds ratios and $95 \%$ confidence intervals $(\mathrm{CI})$ as assessed by univariable and multivariable logistic regression analyses. In these logistic regression models, the dependent variable was the presence of either CKD (defined as eGFR<60 $\mathrm{ml} / \mathrm{min} / 1.73 \mathrm{~m}^{2}$ and/or abnormal albuminuria; $n=37$ ) or cardiovascular complications (defined as presence of ischemic heart disease, ischemic stroke and/or permanent atrial fibrillation; $n=28$ ). In these models LSM was included as dichotomous measure. For the sake of clarity significant $p$-values are highlighted in bold.

Multivariable logistic regression models were adjusted for the following covariates: model 1: age, sex and duration of diabetes; model 2: adjusted for the same covariates of model 1 plus HbA1c, smoking, hypertension (i.e., blood pressure $\geq 140 / 90 \mathrm{mmHg}$ or use of any anti-hypertensive drugs) and dyslipidemia (i.e., plasma LDL cholesterol levels $>100 \mathrm{mg} / \mathrm{dl}$ or use of lipid-lowering drugs); and model 3 : adjusted for the same covariates of model 2 plus body mass index, HOMA-insulin resistance score and plasma hs-CRP concentrations. 
Supplementary Table 1. Association between increasing liver stiffness measurement on vibration-controlled transient elastography (included as continuous measure) and the presence of either chronic kidney disease or cardiovascular complications in patients with non-insulintreated T2DM.

\begin{tabular}{|l|l|l|l|l|}
\hline & \multicolumn{2}{l|}{ Chronic kidney disease } & \multicolumn{2}{l|}{ Cardiovascular complications } \\
\hline & Odds ratio (95\% Cl) & $\boldsymbol{P}$-value & Odds ratio (95\% Cl) & $\boldsymbol{P}$-value \\
\hline Unadjusted model & & & & \\
\hline LSM (for 1-SD increment, i.e. 2.4 kPa) & $1.69(1.15-2.50)$ & $\mathbf{0 . 0 0 8}$ & $1.61(1.08-2.40)$ & $\mathbf{0 . 0 2 1}$ \\
\hline Adjusted model 1 & & & & \\
\hline LSM (for 1-SD increment, i.e. 2.4 kPa) & $1.66(1.11-2.50)$ & $\mathbf{0 . 0 1 5}$ & $1.63(1.07-2.47)$ & $\mathbf{0 . 0 2 2}$ \\
\hline Adjusted model 2 & & & & $\mathbf{0 . 0 1 5}$ \\
\hline LSM (for 1-SD increment, i.e. 2.4 kPa) & $1.72(1.13-2.64)$ & $\mathbf{0 . 0 1 2}$ & $1.79(1.12-2.86)$ & \\
\hline Adjusted model 3 & & & & $\mathbf{0 . 0 3 5}$ \\
\hline LSM (for 1-SD increment, i.e. 2.4 kPa) & $1.68(1.04-2.70)$ & $\mathbf{0 . 0 3 4}$ & $1.73(1.04-2.88)$ & \\
\hline
\end{tabular}

Data are expressed as odds ratios and $95 \%$ confidence intervals $(\mathrm{Cl})$ as assessed by univariable and multivariable logistic regression analyses. In these logistic regression models, the dependent variable was the presence of either CKD (defined as eGFR 60 $\mathrm{ml} / \mathrm{min} / 1.73 \mathrm{~m}^{2}$ and/or abnormal albuminuria; $n=37$ ) or cardiovascular complications (defined as presence of ischemic heart disease, ischemic stroke and/or permanent atrial fibrillation; $n=28$ ). In these models LSM was included as continuous measure (for 1-SD increment). For the sake of clarity significant $p$-values are highlighted in bold.

Multivariable logistic regression models were adjusted for the following covariates: model 1: age, sex and duration of diabetes; model 2: adjusted for the same covariates of model 1 plus HbA1c, smoking, hypertension (i.e., blood pressure $\geq 140 / 90 \mathrm{mmHg}$ or use of any anti-hypertensive drugs) and dyslipidemia (i.e., plasma LDL cholesterol levels $>100 \mathrm{mg} / \mathrm{dl}$ or use of lipid-lowering drugs); and model 3 : adjusted for the same covariates of model 2 plus body mass index, HOMA-insulin resistance score and plasma hs-CRP concentrations. 


\section{FIGURE LEGENDS}

Figure 1. Prevalence of NAFLD and significant/advanced liver fibrosis among patients with non-insulin-treated T2DM without known liver diseases. NAFLD was assessed by ultrasonography, whereas liver fibrosis was measured with the vibration-controlled transient elastography (VCTE, FibroScan ${ }^{\circledR}$ ) using two different thresholds of liver stiffness measurement to identify patients with significant or advanced fibrosis.

Figure 2. Prevalence of chronic kidney dysfunction (defined as eGFR $<60 \mathrm{ml} / \mathrm{min} / 1.73 \mathrm{~m}^{2}$ or abnormal albuminuria) and cardiovascular complications (defined as ischemic heart disease, ischemic stroke or atrial fibrillation) across tertiles of liver stiffness measurement on the vibration-controlled transient elastography (VCTE, FibroScan ${ }^{\circledR}$ ) among patients with non-insulin-treated T2DM without known liver diseases. 